\title{
Comparison among different methods for blood pressure monitoring in rats: literature review
}

\author{
Sidonia BOGDAN ${ }^{1}$, Vlad LUCA ${ }^{1}$, Ciprian OBER ${ }^{1}$, Iulia MELEGA ${ }^{1}$, Cosmin PESTEAN $^{1}$, Razvan CODEA ${ }^{1 *}$, \\ Liviu OANA ${ }^{1}$
}

${ }^{1}$ Department of Preclinical Sciences, University of Agricultural Sciences and Veterinary Medicine, ClujNapoca, Calea Manastur 3-5, 400372, Romania

*corresponding author: razvancodea@yahoo.com

Bulletin UASVM Veterinary Medicine 76(1)/2019

Print ISSN 1843-5270; Electronic ISSN 1843-5378

doi:10.15835/buasvmcn-vm: 2019.0007

\begin{abstract}
Blood pressure is a cardinal vital sign that gives important information about the cardiovascular function and about hemodynamic trends during anaesthesia, in critical ill patients and during experimental procedures (Rehman and Nelson, 2018). Arterial blood pressure can be evaluated by direct technique (arterial catheter) or indirect technique (Doppler or oscillometry). Direct measurement is gold standard for blood pressure measurement, giving accurate beat-to-beat information and also allow collection of blood samples. However, it is more invasive and requires equipment for monitoring and experience to place the arterial catheter (Araghi et al., 2006; Ward and Langton, 2007; Wingfield and Raffe, 2002). Given its importance in directing care, it is essential to measure blood pressure accurately and consistently.

The aim of this article is to present all technique found in literature, with its advantages and disadvantages, with the errors that may occur so that the researchers can have a better knowledge before choosing their technique. For this study we took into consideration articles from literature and speciality books from which we extracted the information reliable for the study. Experimental studies on small laboratory animals, particularly rats are widely used as a model. Three methods are used for recording the blood pressure in rats: tail cuff (indirect technique), intra-arterial catheters (direct technique), and radio telemetry. Each technique has its advantages and disadvantages and each of it may lead to erroneous data if they are improperly performed. The vast ranges of these errors highlight the importance of adhering to appropriate technique when measuring blood pressure.
\end{abstract}

Keywords: blood pressure, rat, invasive, non-invasive techniques

\section{Introduction}

Lee Tyner, a professor and specialist in anesthesia from Mississippi State University once sad that "Death is a late sign of poor perfusion. Patients are not fine one minute and dead the next" which points out the importance of monitoring during a disease, an experiment or under anesthesia to prevent deaths. The primary cause of crisis during or after anesthesia is the failure to notice a problem when it first occurs. The importance of monitoring a patient during anesthesia is to evaluate tissue perfusion which is maintained by the autonomic nervous systems that are depressed during general anesthesia (Faunt, 2008).
Many of in vivo experiments require anesthesia to reduce the pain and to protect the welfare of the experimental animals witch can increase the risk and the number of dead subjects. Although anesthetic safety in veterinary medicine has dramatically increased in the recent decades and the multiple studies presenting the advantages and disadvantages of various anesthetic protocols in experimental animals the number of death related to anesthesia is still major (Tranquilli et al., 2013).

One of the most related ways to evaluate tissue perfusion is by monitoring blood pressure (BP). It drives perfusion by delivery of blood supplies and oxygen to organs as well as tissue beds within the body. 
Constant evaluation of a patient's BP helps avoid serious complications caused by hypertension such as eye, ear, brain and kidney damage or by hypotension which will compromise tissue perfusion to heart, kidney and brain and lead to multiple organ failure. Many diseases and drugs can result in BP changes, so measurement is an important regular diagnostic tool during examination. (Faunt, 2008; Oriana and Scislowics, 2018; Wingfield and Raffe, 2002).

BP is created by the volume of blood ejected by the heart into the vessels and it is influenced by the cardiac output, systemic vascular resistance and blood viscosity. Systolic arterial pressure (SAP) it the measurement of the maximum pressure during ventricular contraction (systole). Diastolic arterial pressure (DAP) is the measurement of the minimum arterial pressure during ventricular relaxation (diastole). Mean arterial pressure (MAP) is the measurement of pressure trough one full cardiac cycle (one heart beat) and it reflects the circulatory status and provides accurate indication of hemodynamic trends. Understanding the role of cardiac output and vascular resistance in MAP values is important because many drugs used affects these in some manner. MAP is determinated by the equation $\mathrm{MAP}=\mathrm{DAP}+(\mathrm{SAP}-\mathrm{DAP}) / 3$. Values of BP are measured in scale called "millimeters of mercury" mmHG (Faunt, 2008; Filipas and Vettorato, 2018; Oriana and Scislowics, 2018; Hentschel, 2008; Wingfield and Raffe, 2002)

BP falls following hypovolemia due to blood or fluid loss, cardiac failure, sepsis, trauma, anesthetic products. High BP occurs in kidney, heart and hepatic deficiency, hormonal imbalances (thyroid variation, adrenal tumors), congenital circulatory problems and some medication. (Filipas and Vettorato, 2018; Wingfield and Raffe, 2002)

There are several means available for recording $\mathrm{BP}$. When a technique to measure BP is chosen, specific issue must be considered like the availability of the equipment, the skills of the person making the measurement, and specific issue related to the species and animal (e.g., size, obesity, temperament) (Ettinger et al., 2017; Rehman and Nelson, 2018).

In experimental studies, the gender and age of the rats are also important. The applicability on rats of different procedures requires modification of the equipment and technique due to small patient size, challenges of restrain, monitoring limitations and increased metabolism. Experimental protocol needs to be taken into consideration; if it is acute or chronic, if it demands continuous monitoring or daily checkups and if it requires anesthesia which can change the values of BP and the hemodynamic trend (De Wolff et al., 1999; Imai et al., 1999; Will, 2003).

During the experiment, all subjects must be closely monitored. Accurate information about function of the cardiovascular system during the protocol can help take prompt actions to correct the imbalances. Administration of anaesthetic drugs, the unconscious, recumbent, immobile state compromise patient homeostasis and is important to monitor the rat in order to detect the specific needs and to maximize the safety of the anaesthetic experience. Anaesthesia and experimental procedure affect the respiratory and cardiovascular system, could cause loss of fluids or induce shock and could compromise the procedure. Also the uncontrolled body temperature during the procedure, manly in anesthetised patients can be critical for the experiment if left unbalanced. Rats under anaesthesia have difficulty regulating their internal body temperature since they have high surface area to body mass ratio. The risk of hypothermia poses great harm to the safety of the animal and cold stress have direct impact cardiac mechanism and function like increase mean arterial pressure and decrease systemic vascular conductance. Maintaining the rats' vital functions within normal range during the anaesthesia is essential for the success of the experiment and by monitoring BP researchers are in direct contact with the homeostasis of subjects (Ordodi et al., 2005; William et al., 2007; Wilson and Crandall, 2011).

The study was based on the data found in literature on PubMed, Research gate, Science direct, speciality books and on first author personal experience with different procedures.

\section{MEASUREMENT TECHNIQUES}

Arterial blood pressure can be evaluated either by direct techniques using invasive procedures with arterial catheters or by indirect techniques applying non-invasive protocols by pulsatile monitoring. The last ones are the most used in experimental protocol because they are easier to apply as they require less technical skills and presents less risk to the patient. Direct BP monitoring it considered the gold standard and provides continuous monitoring of SAP, DAP and MAP (Faunt, 2008; Wingfield and Raffe, 2002).

\section{Invasive blood pressure procedures}

Invasive blood pressure (IBP) procedures register direct arterial BP, but require general anaesthesia in experimental animals for the surgical placement of the arterial catheter the radio-telemetry transmitters. Some of the protocols require general anesthesia for the value recording also. It is important to remember this fact when BP monitoring technique is chosen because anesthetic products used for the chemical restrain of the rats can influence the actual values (Malkoff, 2005; Parasuraman and Raveendran, 2012).

IBP monitoring techniques are the gold standard procedures and reveal the most reliable values if they are correctly applied. BP obtained by artery cannulation is accurate, the values obtained are objective and repeatable which makes this procedure 
suitable for continuous monitoring. The main disadvantages are the fact that require skills to place the catheter or to insert the radio transmitters, and special expensive equipment is needed (Ettinger et al., 2017; Malkoff, 2005; Feng 2008; Wang et al., 2013).

\section{IBP monitoring by artery cannulation}

This technique involves placement of an openlumen catheter in an artery and exteriorizing it at a site inaccessible to the animal, usually in the nape of the neck or at the crown of the head. This procedure demands general anesthesia for the surgical catheter placement. Injectable intra-muscular or intra-peritoneal anesthetic protocols can be used like urethan/ketamin folwed by xylazine/pentobarbital, or just xylazine+ketamine and also mix of injectable and inhalation anesthetic can be used. Products like Isoflurane in combination with tramadol or other analgesics can also be used (Bogdan et al., 2017; Kramer and Kinter, 2003; Meredith, 2015; Ordodi et al., 2005; Parasuraman and Raveendran, 2012). The protocol must be performed in a surgery room or in an aseptic experimental room, a surgery warm table is required and a surgical lamp for a proper view during surgery. General surgical instruments that are used in the procedure should be adapted to the size of the rat; soft, fine, blunt forceps and scissors should be used for the dissection. An arterial catheter made of soft polyethylene tube with a needle inside is required and it should be suitable for the size of the rat. Usually a $22 \mathrm{G}$ to $26 \mathrm{G}$ and 1.5 to $4 \mathrm{~cm}$ long are the appropriate size of the catheters used for carotid, femoral or caudal ventral artery, the most approached arteries in rats. To record BP, the arterial catheter is connected to a pressure transducer through a fluid extender filled with heparinized saline. The extender must be flexible but not elastic otherwise will affect the reading, the pressure must be uniformly transmitted to the transducer. The transducer must be placed at the level of the left atrium and should be calibrated to 0 mmHG pressure. These are then connected to monitor with data acquisition system which collects the signals, amplifies them and convert them into digital numeric value in real time on the screen. The arterial line is also connected to a flushing system consisting of a bag of saline pressurized to $300 \mathrm{mmHg}$ via a flushing device. This will help to keep the catheter clean and without clots which can give erroneous values (Malkoff, 2005; Ordodi et al., 2005; Parasuraman and Raveendran, 2012; Wang et al., 2013; https://www.harvardapparatus. com/rat-catheters.html, https://www.instechlabs. com/products/catheters/rat-carotid).

How to approach the arteries for cannulation?

Carotid artery: Rat is anesthetized and placed in dorsal recumbence and gently restrain the legs to the table with adhesive tape. For a better exposure of the ventral cervical region a syringe or gauzes can be placed under the neck to hyperextend the head. The hair is trimmed and antisepsis is performed. Skin incision is performed with a scalpel on the ventral cervical region a bit to the right/left off the midline of the neck, at the level of the clavicle according to the artery that you want to approach. Small eyelid retractor like blepharostat can be used for a better view. Using a hemostat forceps dissect bluntly the omohyoid muscle longitudinally and expose the carotid artery. It can be identified along with the vagus nerve on either side of the trachea. The vagus nerve and the connective tissue is softly dissected and the artery is isolated with great care not to damage or stimulate the nerve (anatomy in Figure 1). Place a loose tie on the caudal, cardiac end of the artery and a fixed tie on the cervical end of the artery (Figure 2 ). With a micro-dissection hook the artery is softly elevated and bulldog clamp is placed on the cardiac end to block the blood flow during the cannulation. Using a microsurgical scissor, a small incision is performed on the artery. The cannula is inserted in the vessel directing towards the heart and immobilized with a soft needle holder without locking it to damage the vessel or the catheter. The cardiac end loose ligature is now tied on the catheter not too tight to damage the vessel or to affect the blood flow through the catheter. The bulldog clamp can be slowly removed and check if any bleeding occurs meaning that the ligature on the catheter is not stopping the blood flow. If there is no bleeding the clamp can be removed. If there is bleeding the artery should be clamped again. Another ligature to imobilise the walls of the artery to the catheter is placed or adhesive glue can be used at the cannulation site. It is important to have a good fixation, otherwise later bleeding can occur or expulsion of the catheter due to high BP. Skin can be sutured with simple or continuous pattern or even steel clips can be applied. The catheter is connected to pressure transducer and values can be recorded. Catheter is flushed with heparinized saline and can be left in place for more days to record BP. Rats can be kept sedated for the monitoring sessions or they can be awaken and monitor values in conscious rats. To protect the catheter, long extender filed with heparinized saline must be placed between the catheter and the transducer to allow the movement of the rat in the cage. Also a three way valve must be placed on the line to flush the cannula with 0.05 heparinized saline every hour for 24h (Bogdan et al., 2017; Feng 2015; Krinke, 2000; Parasuraman and Raveendran, 2012).

Femoral artery: Anesthetised rats are placed in dorsal recumbency and the inguinal region is surgically prepared. Incision of the skin of $1-1.5 \mathrm{~cm}$ is performed 


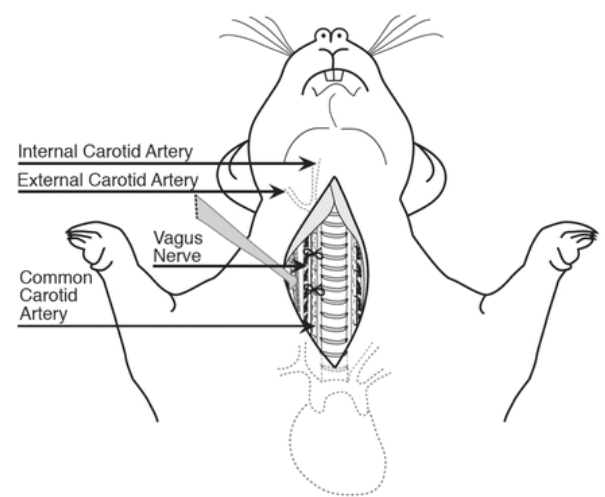

Figure 1. Approach of the right carotid artery

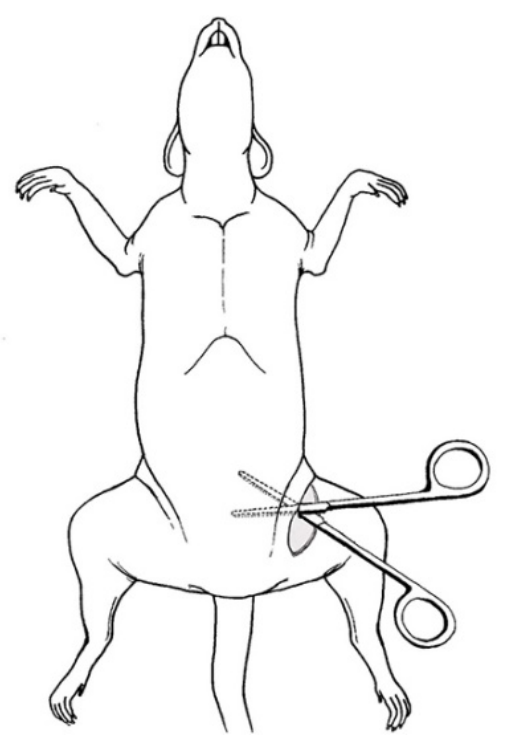

Figure 4. Skin incision for femoral artery approche (Jespersen et al., 2012)

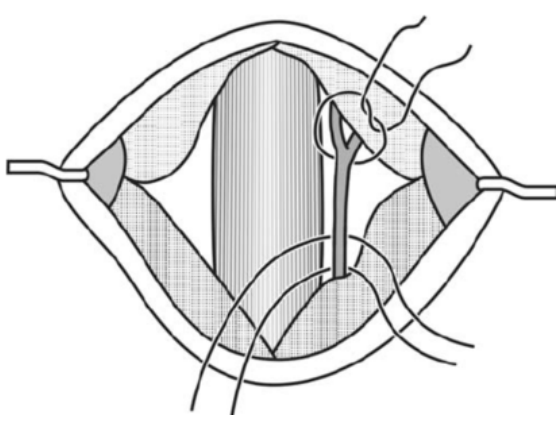

Figure 2. Carotid artery ligation (left carotid image) (Huetteman and Bogie, 2009)

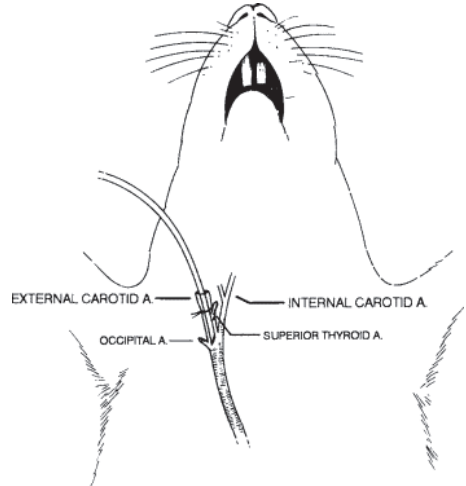

Figure 3. Securing the catheter

with the distal ligature
A

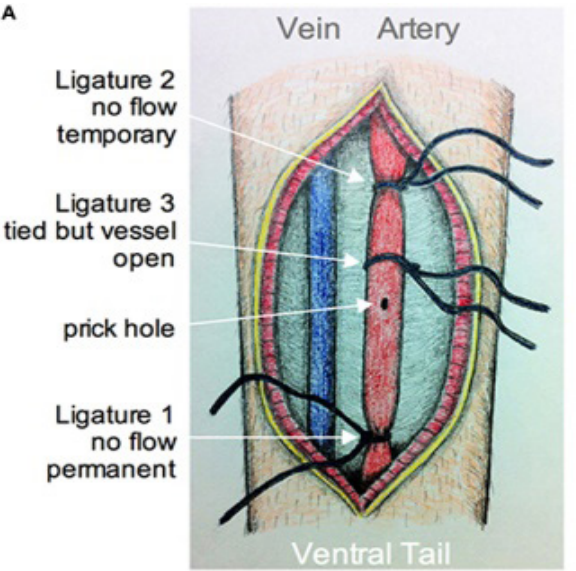

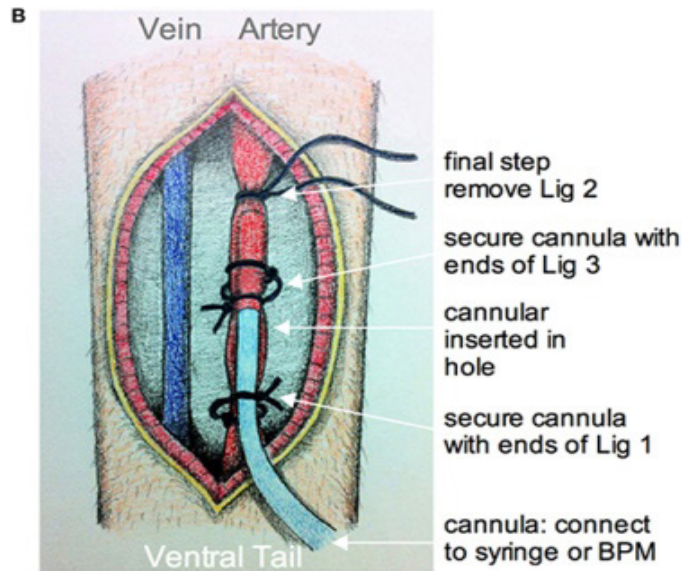

Figure 7. Approach of the caudal ventral artery (Tran and Gordon, 2015) 
perpendicular to the limb and parallel to the abdominal line in the inguinal region (Figure 4). These will access the proximal segment of the femoral artery and leave the distal part of the limb for catheter securing. Retractors must be placed on the skin for a better view. Same steps must be followed from now: 2 ligatures must be placed, one loose on the cardiac end and one tight on the opposite part, the proximal part which will enlarge the artery by obliterating the blood flow. Space is limited in this region for a better approach the artery is suspended with the wire from the second ligature, carefully without tearing the artery. Next step is to make a small incision on the artery between the 2 ligatures and to insert the catheter (Figure 6). The catheter is inserted around $2 \mathrm{~cm}$ until it reaches the abdominal aorta and is fixed with the proximal ligature. The size of the catheter should be adapted, a 24G may be big for a small rat and a $26 \mathrm{G}$ may be proper for femoral aorta. Finish the procedure by suturing the skin (Jespersen et al., 2012; Lin et al., 2019).

Caudal ventral artery: Rats are anesthetized and placed in dorsal recumbency. Hair from the base of the tail is trimmed and the region is antiseptic prepared. On the ventral surface, near the base of the tail in the middle line the skin is incised. Artery is easily visualized and soft dissection is performed. There is a lack of connective and soft tissue in this region so care must be applied not to tear the artery during isolation. The next steps are the same as the one presented in the carotid and femoral approach (Figure 7)(Kregel et al., 1988; Wang et al., 2013).

The monitoring of BP can be done under sedation for acute experiments. Isoflurane can be used for prolonged sedation and the effect on BP is minimal if low concentration is used. Artery cannulation allows researchers to monitor live BP for longer periods even in awaken rats. For this, the catheters must be tunneled under the skin and fixed on the surface of the skin on the dorsal cervical region. The proximal ligature which has the role to imobilise the catheter inside the artey must be changed surgically every 7-10 days. Care must be taken on al steps, bleeding and severe hemorrhage can occur, the catheter can obliterate so regular flushing must be done. Great importance must be given to the catheter preparing, maintenance and the filling with heparinized saline of all the tubes that are connected to the catheter. It is momentous not to have air bubbles and twists on the catheter and on the tubing; otherwise the values recorded will not be relevant; air injected in the arterial patch is forbidden (Feng, 2015; Jespersen et al., 2012; Parasuraman and Raveendran, 2012; William et al., 2007)

\section{IBP monitoring by radio-telemetry}

This is a highly invasive surgical procedure, but very reliable. Telemetry involves the implantation of a radio transmitter in the rodent's body. This technique is validated and has excellent correlation with direct $\mathrm{BP}$ monitoring by artery cannulation. The present system has the advantage that radio telemetry implants have the ability to measure BP continuously in free-moving rats. Nowadays, measurement of BP by radiotelemetry has been described and validated for many laboratory animal species, including rats and mice. (Kramer and Kinter 2003; Malkoff, 2005).

The basic rat procedure involves advancing a blood pressure catheter into the abdominal aorta which is connected to a radio transmitting device which is placed in the peritoneal cavity. The technique takes about 30 to 40 minutes and requires general anesthesia. Rat must be placed in dorsal recumbency and the abdominal region prepared for surgery. The incision is made on the middle line for about 3 to $4 \mathrm{~cm}$ including the skin and the linea alba of the muscle layer separately. Gently isolate the intestines using moistened cotton applicators; retract the intestines using moistened gauze sponges to provide a good visualization of the aorta between left renal vein and artery cranially and the iliac bifurcation caudally (Figure 7). The descending aorta is located along the dorsal body wall. The aorta is carefully prepared, the surrounding fat and connective tissue is dissected using cotton tip applicators. Two temporary occlusion sutures are placed with caution around the aorta, one after the bifurcation of the left renal artery and one before the iliac arteries bifurcation. Traction the suture to block the blood flow between the sutures, insert a needle with the tip in $90^{\circ}$ to perforate the aorta before the caudal suture and also use it as a retractor (Figure 8). Insert the special catheter until its tip is in contact with the cranial oclusion suture. Clear the blood from the catheter area using a cotton tip applicator and apply a drop of adhesive on the catheter and around the cunture site. Let it dry for 30 seconds and then slowly release the cranial oclusion. If bleeding occurs repeat the last step use additional glue after the site is dry out of blood. The catheter is imobilised with a fiber patch secured with adesive glue. Reposition the intestines in their original position and place on top of the organs the device which is conected to the cateter Next step is to close the abdomen wall with nonresorbable suture and imobilise in this suture the device. Close the skin and recover the rat from the aneshesia. Analgesics must be administered for few days. After the surgical technique each rat must be allowed to recover for 7 days before pressure measurements and be placed in individual boxes. On the day of recording, conscious unrestrained rats are placed in the box for blood pressure monitoring and let to acclimate with the new environment for 30 minutes before recording values (Huetteman and Bogie, 2009, Malkoff, 2005; Oudot et al., 2009).

The advantage of implantable radio telemetry is the ability to measure blood pressure continuously 


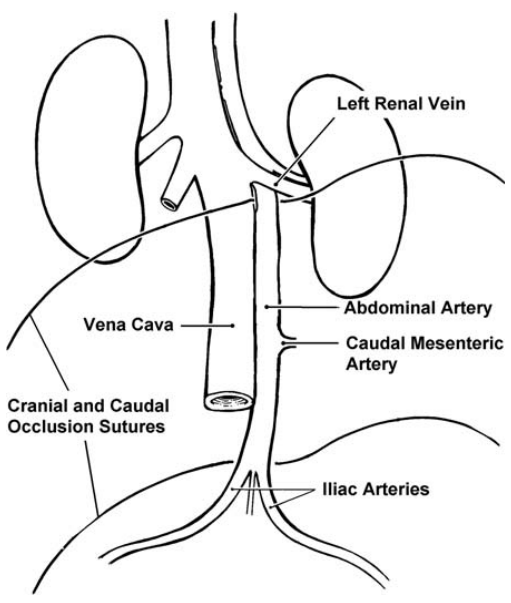

Figure 8. Approach of the abdominal aorta and placement of the temporary occlusion (Huetteman and Bogie, 2009)

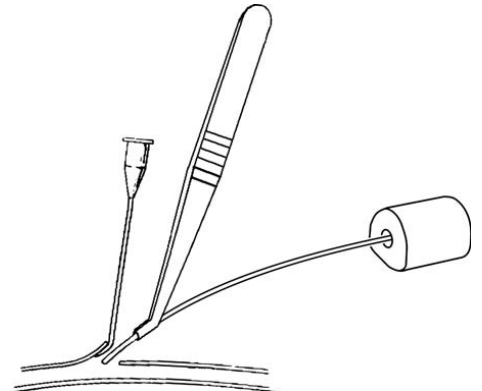

Figure 9. Catheter insertion in the aorta (Huetteman and Bogie, 2009)

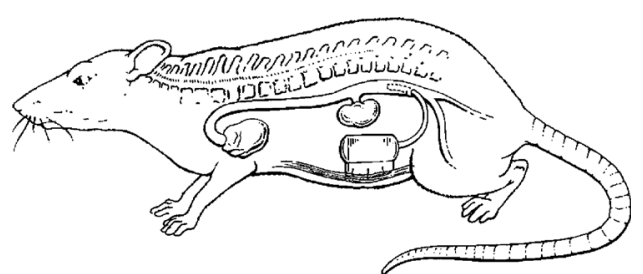

Figure 10. Position of the radio telemetric device in the abdominal cavity (Huetteman and Bogie, 2009) in free-moving laboratory animals. This procedure allows the scientist to record mean arterial pressure, systolic and diastolic arterial pressure, pulse pressure, heart rate and temperature. The disadvantages of this techinque are many. High risk mortality during the surgery, increased level of stress caused by the weight of the transmitters and by lack of social interaction (one animal/cage). Another disadvantage is the high cost for the equipment, for the transmitters and human resources (Malkoff, 2005).

\section{Non-invasive blood pressure procedures}

Non-invasive or indirect blood pressure procedures rely on a system that detects blood flow under or past a pressurized cuff. These methods consist of placing a cuff on the animals' arteries to occlude the blood flow and upon deflation the sensors, which are placed distal to the occlusion can monitor blood pressure. There are three types of sensor technologies used: photoplethysmograpy, piezoplenthysmography and volume pressure recording. The periphery artery approached in rats is the ventral caudal artery and these methods use a tail cuff special designed for the size of rat (Durham and Edward, 2005; Malkoff, 2005).

A fundamental principle of non-invasive BP measurement is that the pressure measured is inside the cuff, not in the vessel itself. The cuff it is important to be adapted to the size of the rat tail. It should be $40 \%$ of the circumference of the tail. If the cuff is larger, the values will be lower and if the cuff is thinner the values recorder will be higher. The cuff should be placed at the base of the tail and it is important to keep it at the level of the heart. Hand restraining is not suitable for recording $\mathrm{BP}$, the level of stress is high, special holders in different sizes are used to monitor BP in conscious rats. Chemical restrain should be avoided, as it often alters results (Durham and Edward, 2005; Faunt, 2008; Ng and Small, 1993; Wingfield and Raffe, 2002).

Animal preparation and acclimatisation is also important. Recording should be performed in a quiet and comfortable environment with no distractions (other animals, busy activity, odorous smells caused by perfume, chemical cleaners) and only after the rat was acclimatized. Proper animal handling is critical to have accurate blood pressure measurements. A nervous, stressed animal may have diminished circulation in the tail, tachycardia can be suggestive of an increased level of stress. Rodents can be trained easily in about three days, 15 minutes each day before the beginning of your experiment. The rat should enter in the holder freely and after he is in the nose cone can be adjusted so the animal is sitting comfortable, but it should not be able to move excessively. The nose should protrude through the front of the cone to allow breathing. If the cone is dark it reduces the stress by limiting the view to the environment. The animal should never have its head flexed sideways or the body compressed against the back hatch. The rat should not move during the recording; any motion of the hose may be interpreted as fluctuation occurring during the blood pressure reading. A proper size of animal holder is essential for proper blood pressure measurement. (Burkitt Creedon, 2012; Malkoff, 2005).

The first part in monitoring BP with non-invasive techniques is similar. The first step is to put the rat in the holder, let it enter freely, carefully immobilize the tail outside and adjust the nose cone in front to limit the movement of the rat. Let the rat acclimatize for 5 


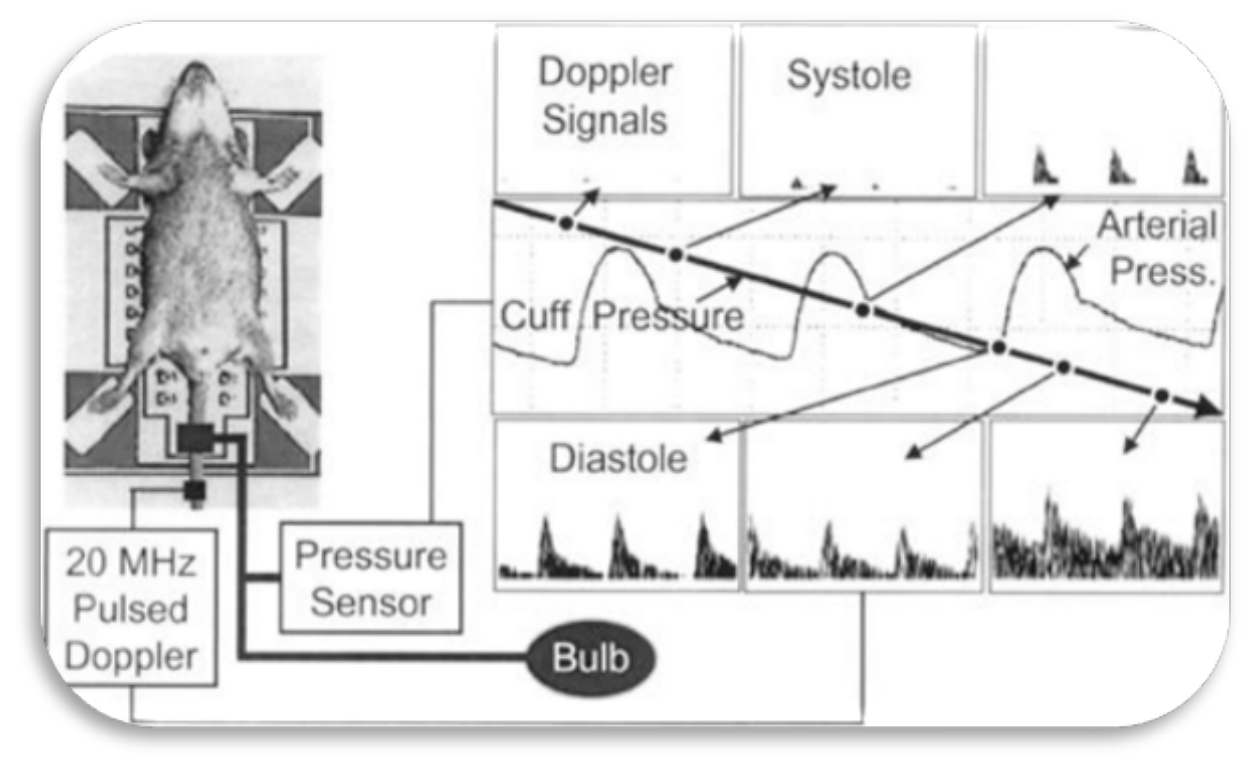

Figure 11. Illustration of how systolic and diastolic blood pressure can be measured using a pressure cuff and a Doppler cuff placed on the tail of the rat (Hartley et al., 2002)

minutes, without touching it or making noise. Then select the proper cuff for the tail and place it at the base of the tail. It is important that the rat to be relaxed with the tail ventrally so the cuff will be at the level of the hart. It is also important to monitor temperature before and during the monitoring session. The animal must have adequate blood flow in the tail to produce pressure signals. Anesthetized rats may have lower body temperature and it is important to correct the body temperature before recording (Malkoff, 2005).

Then the recording sensor is applied distally to the cuff, usually $2 \mathrm{~mm}$ distance, according to the procedure used. The cuff and the sensors are connected to monitors and systems which record the values obtained and display them.

\section{Piezoplethismography systems}

The Doppler system has been shown to be effective in measuring SAP. The method is similar to the concept used in humans, were the nurse listens with a stethoscope to the vibration of the arterial wall caused by the turbulent flow known as Korotkoff sounds. The sound is produced by the first blood flow through the artery after slowly releasing the pressure from the cuff. The cuff is attached to a manometer or a mercury sphygmomanometer to display the pressure in millimetres of mercury. In rats the Korotkoff sounds are inaudible and Doppler piezoelectric ceramic crystals are used to detect blood flow. It can detect even low values in case of hypotension or vascular insufficiency (Durham and Edward, 2005; Ward and Langton, 2007).
When the rat is ready, apply acoustic gel on the Doppler crystal and attach it on the ventral side of the tail distally to the cuff. Doppler is turned on to hear the pulsatile whooshing sound of the blood flow through the artery. Then the cuff is inflated with $20 \mathrm{mmHg}$ higher than expected to occlude the artery pressure; now the arterial pulsations sound should stop. The cuff is slowly deflated and then recorded the pressure from the manometer at the moment of the first sound of the arterial pulse. This value represents the systolic blood pressure; the Doppler method has been shown to be a very poor estimator of DAP. Some researchers prefer to record BP under sedation. The system is described in Figure 11 (Burkitt Creedon, 2012; Ettinger et al., 2017; Kregel et al., 1988).

This crystal is very sensitive and can detect signals even from mice with high pulse rates. The skin pigmentation does not influence the piezoelectric crystal affinity; values can be inaccurate if movement occurs, if gel is insufficient, if cuff is fast deflated or is too much inflated, if rat's temperature is modified or if rats are under sedation. The system was adapted and tested in freely moving rats, but values obtained were higher comparing with the restrained or anesthetised rats (Erken et al., 2013; Imai et al., 1999; Malkoff, 2005).

\section{Volume Pressure systems (VPS)}

The Volume pressure recording systems (Figure 12, Figure 13) uses a specially design differential transducer to measure six parameters from the blood in the tail, non-invasively. It can register SAP, DAP, MAP, heart rate, tail blood volume and tail blood flow. According 


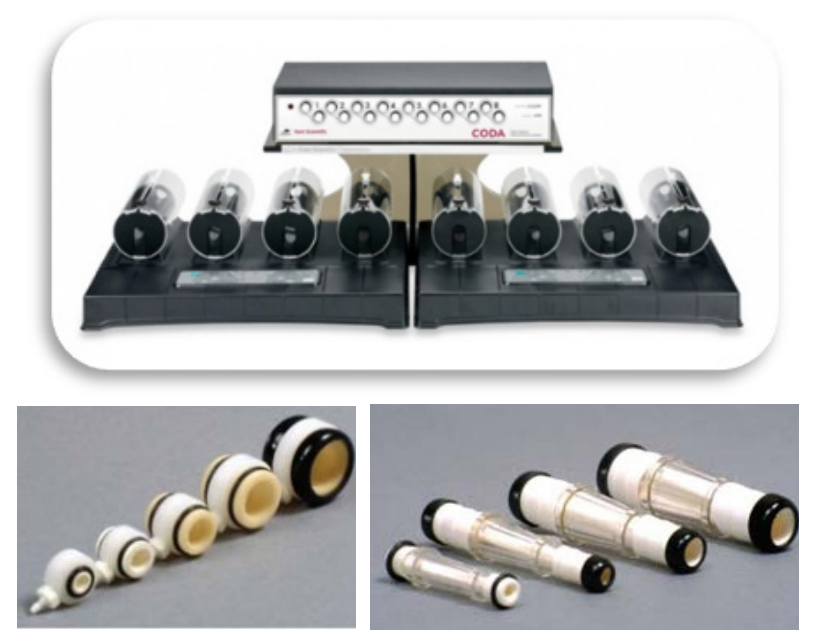

Figure 12. Volume pressure recording system by CODA for 8 rats https://www.kentscientific.com/ products/coda-high-throughput-system/

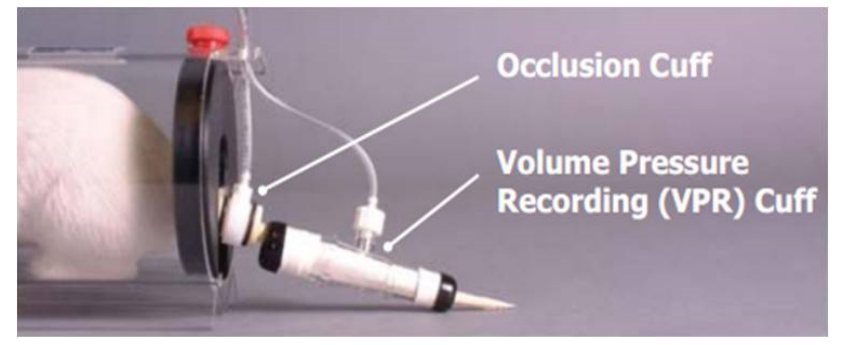

Figure 13. A: pressure cuff, B: tail cuff, C How system is applied on the rat tail http:// www.itsbio.co.kr/main/gmb_board_view. php?page $=1 \&$ no $=444 \&$ search $=\&$ page_no $=1$

design with the system (https://www.wpi-europe. com/products/animal-physiology/blood-pressuremeasurement/ii-mrbp-r.aspx and https://www.iitcinc. com/3man.html). By using a light-based technology, it records the first appearance of the pulse while deflating the occlusion cuff (Jin et al., 2013; Malkoff, 2005; Patten and Abeywardena, 2017).

Also these systems have their disadvantages. They can be inaccurate since the reading is based solely on the amplitude of a single pulse and cannot measure SAB on the heartbeat. If the system is not correctly used, the values can be false because measurement can be influenced by ambient light, movement of the rat and also by the skin pigmentation. The light sensor can cause tail burns from close contact and prolonged exposure. It is mandatory to test the system, to understand the principle of functioning and to check its limits before using it (Malkoff, 2005)

A critical part of the process is the placement of the inflation and detection cuff onto the test subject. Most of the systems have the tail cuff placed proximal and the sensor distally which includes manipulation of the tail, others combined both into one single tail cuff sensor. This technology eliminates the need for restraining the animal's tail and creating undue stress (NIBP Multi Chanel Blood Pressure System design by IITC Inc. Life Science) (Jin et al., 2013; Whitesall et al., 2004).

For all three systems presented above there are some aspects that need to be taken in consideration while recording. BP can be influenced by many factors, and the values obtained may not be reliable for the study. BP may vary in the first measurement, if the temperature is different (of the host or of the 


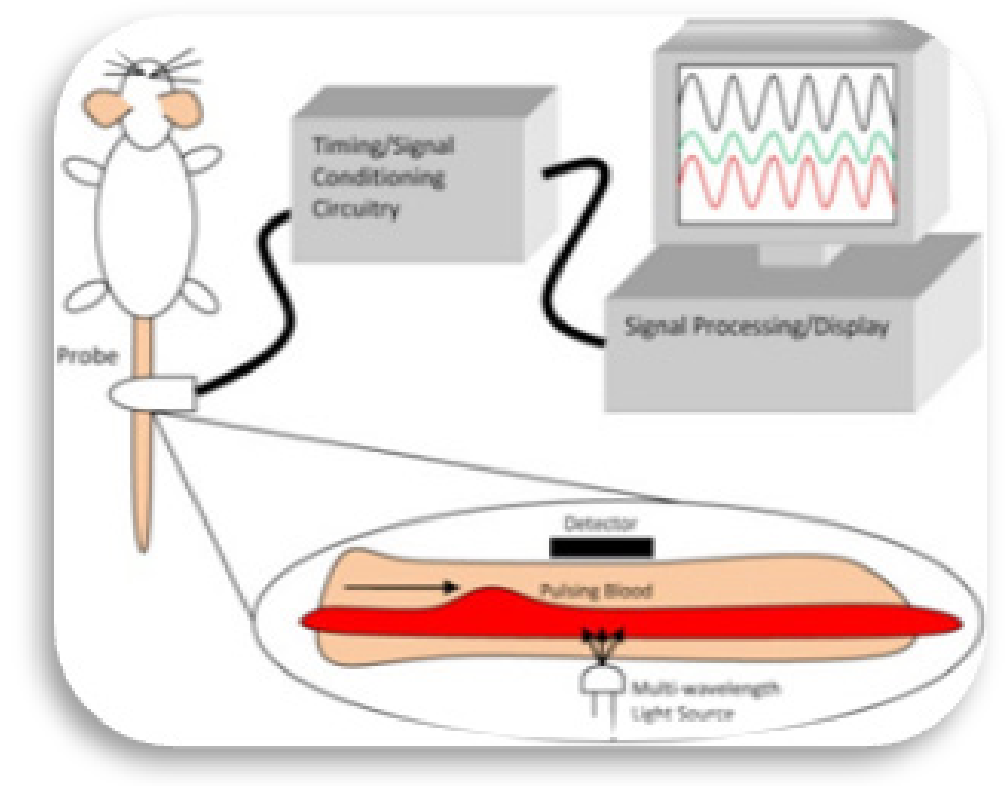

Figure 14. Schematic, the photoelectric sensor coupled to rats' tail and diagram of the examined pulsatile arterial blood (Michalak et al., 2010)

environment) and can be severely influenced by stress, noise and handling. It is also important to measure at the same time each day because BP varies according to the time of day (Howatt et al., 2017).

Tail temperature is important. To measure the temperature an infrared thermometer can be used to check the temperature of rat's tails. The suggested ideal tail temperature for the blood pressure analysis for most of the techniques is $33-35^{\circ} \mathrm{C}$ some even $36-40^{\circ} \mathrm{C}$, but these systems can adjust to lower temperature also. To maintain the temperature within limits warming platforms or infrared lamps can be used and room temperature should be $20^{\circ} \mathrm{C}$ or above. If the rats tail is too cold the blood flow in the tail will be reduced and $\mathrm{BP}$ will not be read. Conversely, if the warming platform, heating lamp or hot air heating chambers are too hot rats will increase the respiratory rate and stress levels can be high, and may lead to severe dehydration or even death (Malkoff, 2005).

\section{Discussions}

Many studies reveal those tail cuffs methods can be accurate, consistent and applied in studies of awaken and anesthetized rats. The errors may occur if the rats are not handled properly and if rats are not trained. Steps must be performed accordingly and attention must be paid to the external factors like noise, temperature, odours, technician, etc. Many articles presented the variation of $\mathrm{BP}$ and heart rate during and after restrain and differences between the values obtained with direct and indirect methods.
Studies revealed that the tail-cuff technique using volume pressure recording underestimates the core BP changes that occur simultaneously during the restraint and measurement phases. Researchers proved that measurements between the 2 techniques are similar when tail-cuff readings are compared with telemetry readings in the not-disturbed animals. This suggests that not only the stress of handling is changing the central BP by changing the heart rate, but also values may vary from one technique to another according to rat response to stress. This needs to be taken in consideration by the researcher, to choose the steps and time of recording wisely and to pay attention to the stress factors in the surroundings while recording (Wilde et al., 2017; Whitesall et al., 2004).

Comfort is important while recording BP in awaken rats. The technique is dependent on maintaining a minimum amount of blood flow in the tail and any physiological, pharmacological or environmental factors that affects tail blood flow will affect the BP measurement. Temperature may influence the values recorded by NIPB procedures by reducing the blood flow to the tail which can lead to inaccurate data. Even when minimal external warming is used, the combination of restraint and warming may lead to significant increases in core body temperature (BuÑag and Butterfield, 1982; Kramer and Remie, 2005). One of the mechanisms of thermoregulation is by vasoconstriction to block losing heat or by vasodilation to lose heat. Since the tail is the only part of a rat body without fur, the vascularization of the tail is directly influenced by the ambient and 
central temperature. Body temperature may decrees faster in male rats, loos of heat may be due to the large genital area were the testicle are without fur. Indirect procedure uses the tail to register BP so maintaining rat's constant temperature is important. ITC Life Science Instruments, renowned manufacturer of these systems indicate that ambient temperature should not be under $20^{\circ} \mathrm{C}$ and restrain chamber should be warm. Systems use warm blankets, infrared lamps and warming chambers. NIBP Multi Channel Blood Pressure Systems created by IITC Life Science Instruments uses warm chambers and seems to reduce the thermal stress better than the other two methods (http://www.iitcinc. com/Mouseratbloodpressure.html). Heat and also cold stress have direct impact on many control mechanism of cardiovascular function. Stroke volume, diastolic compliance, systolic function is changed by alteration of hearts inotropism and by changes in peripheral vasodilation/vasoconstriction (Wilson and Crandall, 2011).

Many researchers chose Doppler technique. One of the best part is the accuracy of the piezoelectric ceramic crystal witch can detect blood flow in extremely small arteries and can be used in very small laboratory animals like mice of 18 grams. Reddy validated this method in 2003 by comparing it with direct BP measurements by carotid catheterization in mice between 18-38g. Values obtained simultaneously by both procedures were linear in high and also low values. From $148 \mathrm{mmHg}$ systolic pressure measurements ranged from 58 to 250 $\mathrm{mmHg}$ he obtained a linear relation between tail-cuff method and catheter pressures (Reddy et al., 2003).

From indirect procedures the tail cuff volume pressure systems seem to be the most accurate and have the highest degree of correlation with direct techniques witch are gold standard for BP monitoring. A study performed in mice validated this by measuring in real time SAP with VPS and radio-telemetry devices on awaken animals. The data presented demonstrate that VPS measurements of SBP generally agree with simultaneous telemetry measurements across the physiological blood pressure range in mice (Feng, 2008).

Most of the experimental studies that are performed on lab animals are extrapolated in humans. Further studies will confirm if the hypothesis from experiments can be accepted in human medicine. It is important when choosing an experimental study or a technique to be reliable and comparable with human pathologies. Theodore Kurtz has dedicated his research on therapeutic hypotension and tested all the techniques in many studies, and also presented some recommendation in $\mathrm{BP}$ in humans and experimental studies. His recommendation for NIBP are for studies involving frank systolic hypertension, when working with substantial group differences in systolic BP or substantial changes in systolic BP over time. Other indications are for experiments with large numbers of animals. These techniques are not feasible for quantifying relations between BP and other variables (target organ damage) or for studying BP-independent effect of any intervention or variable (drugs, diet, and genotype). Also, subtle forms of hypertension or light changes or variability in BP cannot be correctly detected with indirect techniques. These procedures do not have accuracy in recording DBP or MAP so researches should focus in SAP wish is very accurate. Tail cuff methods using systems may be expensive but they are very costeffective for large scale, high throughput screening (Kurtz et al., 2005).

Both radio telemetry and tail-cuff determinations of systolic pressure have strengths and weaknesses. There are limitations to measuring blood pressure by either tail-cuff or telemetry and careful consideration should be given to which method to use for a specific experimental design.

Radio telemetry measurement of intravascular blood pressure requires an invasive surgical procedure associated with operative and postoperative morbidity and mortality. Morbidity is associated with the initial surgical implantation of the transmitter, with the surgery that is performed later for battery replacement and with increased stress levels. The last is caused by the several invasive surgical procedures, by weight of the transmitters and by the social limitation (unable to interact with others due to one animal per cage). However, the advantages of telemetry device is the ability to record continuously and detailed the cardiovascular performance in a circadian pattern or a transient treatment responses (Kramer and Kinter, 2003; Malkoff, 2005). After the surgical implantation a recovery period is necessary for about one week. One study recorded daily blood pressure via radio telemetry devices and observed the return of circadian patterns begins to appear within a week, showing a 10 PM to 6 AM peak and a 10 AM to 6 PM valley in both blood pressure and heart rate.

Circadian pattern is important when measuring BP for longer experiments because the values are not constant all day long. If NIBP technique is chosen, time of the day can influence the values recorded. It is important to exclude erroneous values for different time of the days and BP recording should be at the same time every day. Because individual tail-cuff measurements are taken at a discrete moment in time, one single measurement could be greatly affected by these indirect factors, interpretation of the experimental data could also be affected. This error is excluded when continuous direct BP procedures are chosen. (Feng, 2008).

When radio telemetry is taken into consideration for BP recording the mass of the implanted device is equally important. Significant physiological stress 
effects, distress and discomfort can be caused in rats by adding mass to the animal body. Increasing the mass in abdominal viscera can compromise diaphragmatic movement and alter breathing pattern (Braga and Burmeister, 2011). Therefore, telemetric devices should be as lightweight as possible, the device must avoid or minimize any compromise of normal physiologic function or welfare of the animal. Telemetric device can be implanted only in rats over 175g (Braga and Prabhakar, 2009). They reported the use of a telemetric device for mice in rats. By implanting a smaller transmitter the technique is suitable for cases with limited space in the abdominal cavity, like during pregnancy. The values obtained for BP and heart rate were similar to those obtained by using a larger telemetric device developed for rats.

In a review about telemetry devices by Kramer and Kinter (2003) presents numerous of studies validating this technique. He also suggested that it is not feasible to conduct simultaneous measurements using telemetry and conventional technologies during transmitter implantation in rodents, due to the small size of their blood vessels. He recommends parallel study groups for each procedure.

One of the main advantages of using telemetry device is that long term studies can benefit from cardiovascular monitoring from weeks to months. Any pathology that involves BP or cardiac function changes can benefit from this procedure of monitoring. Also, medical effects from vasoactive agents can be observed or any drug safety can be monitored with this technique (Alam et al., 2016; Behr-Roussel et al., 2008; Brockway et al., 1991; Oudot et al., 2009). Radiotelemetry was used in a study were pharmacological response over cardiac effects were investigated with different drugs like remifentanil, dopamine, morphine, atenolol, itraconazol, etc. administered in different routes like orally, subcutaneously and intravenous (Accardi et al., 2016).

Arterial catheterization was used in multiple studies acute and chronic also. Through this method multiple blood samples with minimal restrain can be collected to measure hormones or different parameters can be evaluated frequently. Also it is very suitable for pharmacokinetics studies. Multiple arteryes may be approached; carotid, femoral and caudal ventral artery. (Jespersen et al., 2012; Parasuraman and Raveendran, 2012).

It is important to take in consideration all the factors when choosing the BP procedure. There may be differences even between the artery chosen. One study by Wang et al. (2013) showed differences between caudal ventral artery and femoral artery catheterization and errors that may occur while recording. Baseline values on healthy rats reveal higher values by femoral catheterization comparing with caudal ventral artery.
The difference between those two was stable in 40 recording series. Changes in BP were measured in different hemodynamic trends also. After bloodletting when subjects were hypovolemic the values recorded has changed because and also the differences between artery recordings. Now, the differences between tow arteries was less because vasoconstriction occur in caudal ventral artery and MAP values were now overestimated (Wang et al., 2013). This is a proof that in the same hemodynamic trend values can be different and interpretation may be erroneous if unsuitable technique is chosen for an experiment.

A meticulous study on BP measurement in mice using exteriorized catheters showed a failure rate of $50 \%$ by the end of the fourth week after implantation. Keeping the catheters intact in the arteries for longitudinal studies is challenging for utilization in high throughput screening. Exteriorized catheters may be a source of stress for rats. In a study where researchers compared values of BP obtained from catheters with radiotelemetry observed significantly higher levels of systolic and mean BP (both $+17 \mathrm{mmHg}$ ) from catheter recodings. Although exteriorized catheters do allow for blood pressure to be measured continuously, decreasing catheter patency is a problem that usually limits the duration of reliable recordings. (Bazil et al., 1993; Braga and Prabhakar, 2009; Kramer and Remie, 2005; Reddy et al., 2003).

Catheters are an important factor in recording reliable data. This technique has more failure in mice comparing with rats, because the small size of the catheters. In rats is important to fit the catheter to the vascular size and must be correlated with the size of the animal. Rats suitable for this technique should be greater than 200 grams. If the smaller catheter is used it will result in increasing blood flow resistance (Feng, 2015).

If catheterization with telemetry are compared, both procedures are invasive, require anesthesia, have the surgical risk, but both can be reliable for $\mathrm{BP}$ recordings if the technique is correctly applied. Catheterization however has the benefit of low operation cost, offers the possibility to administer drugs easily, includes less" setup" and collection of blood sample in freely moving animals.

As an alternative to radiotelemetry and arterial catheters, tail-cuff measurement of blood pressure avoids general anesthesia and surgery, postoperative stress, and behavioural stress of isolation required by direct techniques. However, changes in the hour interval used for recording, ambient conditions, operator handling of each animal, or subtle behavioural differences between groups, strains or individual animals can introduce experimental variability to the measurement (Feng, 2008). 
Table 1. Recommendations, advantages and disadvantages between invasive and NIBP techniques

\begin{tabular}{|c|c|}
\hline NIBP techniques & Invasive $\mathrm{BP}$ techniques \\
\hline $\begin{array}{l}\text { Recommendations for screening: } \\
\text { - } \quad \text { systolic hypertension } \\
\text { - } \quad \text { substantial groups } \\
\text { substantial variability in BP values } \\
\text { - } \quad \text { between individuals and groups } \\
\text { long term experiments }\end{array}$ & $\begin{array}{l}\text { Recommendations for screening: } \\
\text { - } \quad \text { identifying intermittent or subtle changes in BP } \\
\text { - } \quad \text { quantification relationships between } \\
\text { BP and other variables } \\
\text { - } \quad \text { BP effect of different interventions, drugs, diet } \\
\text { - BP continuously }\end{array}$ \\
\hline $\begin{array}{ll}\text { Advantages } \\
\text { - } & \text { Easy to apply } \\
\text { - } & \text { Noninvasive } \\
\text { - } & \text { Stress is reduced for the rat } \\
\text { - } & \text { Less expensive equipment }\end{array}$ & $\begin{array}{l}\text { Advantages } \\
\text { - Can record BP values in unrestrained } \\
\text { rats (telemetry devices) } \\
\text { - } \quad \text { The most precise values of B } \\
\text { - } \quad \text { Cecord values over a day cycle } \\
\text { - } \quad \text { Reduces the variability caused by outside factors } \\
\text { - } \quad \text { Have direct access to arterial line to } \\
\quad \text { deliver drugs (artery cannulation) }\end{array}$ \\
\hline
\end{tabular}

Disadvantages

- Cannot detect variabilities

- Cannot be applied on unrestrained animals

- Cannot record values continuously

- Require training before the screening starts

- Not well suited for measuring DAP

- Some may require sedation
Disadvantages

- Require surgery and surgical skils

- Require anesthesia

- Expensive and invasive

- High level of stress for the rat with increase mortality

- Not suitable for screening large number of rats
Blood pressure is influenced by many factors. Any imbalances in cardiovascular system will change $\mathrm{BP}$ values. It is affected by cardiac function which is influenced by cardiac output which in turn is influenced by stroke volume, preload, afterload, contractility and heart rate. BP is also influenced by the vessel system (contractility) and by volemia. It is important when BP is monitored to understand at which level the cardio vascular system was changed and why BP is modified. It is mandatory to understand the correct response of the body to the pathology followed in the experiment or to value the correct effect of products tested and the pharmacological effect.

There are some special catheters with sensors that can be inserted in the ventricles to evaluate more precise ventricular blood pressure and the heart function. They are used in many experiments for drug testing, vary between different types of hearts (e.g., heart failure models, hypertrophy and infarction). Ventricular pressure-volume systems have become well established as the most rigorous and comprehensive ways to assess intact heart function. The surgical technique is described in detail by Pacher et al. (2008).

Recommendations for the use of direct methods for measuring BP in rats experiment are for quantifying the magnitude of hypertension or of changes in $\mathrm{BP}$ and quantifying relationships between BP and other variables (target organ damage). Simultaneous recording of BP and other parameters such as organ blood flow can be obtained in real-time to examine the real-time perfusion pressure flow relationships in conscious unrestrained animals before and after experimental interventions. IBP techinques are suitable for studying BP-independent and BP-dependent effects of different interventions or variables (eg, drugs, diet, genotype, etc), particularly when small BP differences may have significant physiological or pathophysiological impact. Intermittent or subtle forms of hypertension or changes or variability in BP can be identified because of the possibility to measure continuously, in unrestrained animals over a long period. The high-fidelity BP phenotyping that 
is achievable with BP radiotelemetry and with direct measurements through external catheter systems has also provided an invaluable tool for the investigation of genetic determinants of BP regulation (Kurtz et al., 2005).

There is not such a technique witch can be called the best. But each technique is suitable for different experiments and different procedures. Because the principle of measuring $\mathrm{BP}$ is different for each technique it is important to take in consideration everything that involves the procedure while interpreting the values.

Noninvasive procedures are less expensive that invasive techniques but, may not be as precise as them. Direct BP recording is very accurate but, the risk that involves the surgical technique can influence the evolution of the experiment. Before you decided that a direct procedure is suitable for your experiment you need to be sure there is someone in your team with micro-surgical experience and a testing group is mandatory. Indirect techniques can be applied in long studies from weeks to months but are not suitable for continuous monitoring. Repeatable values may be recorded over one day, but, not live continuous values. This can be obtaining only by direct procedures. Invasive blood pressure recording systems are gold standard for arterial pressure measuring. All invasive techniques require anesthesia for catheter or radiotelemeter device implantation which may put the rats at risk. Some of the procedures with arterial catheter may demand anesthesia during the monitor session also. This may influence the real BP values. Prolonged anesthesia in rats is risky, because of the small size, a lack of possibility to monitor and sustain the animal in hemodynamic imbalances.

All of the above must be taken in consideration when choosing the proper procedure. How long is the experiment, acute or chronic? What it is expected from the values of BP to reveal? It is wanted to detect any light change or dynamic values in time? The experiment is critical and continuous measurement is necessary, but anesthesia can be risk for the experiment? Many aspects must be considered when you choose the procedure, but other question is mandatory: Want is the purpose of recording blood pressure and what is targeted by the monitoring? If you answer to this and understand how each technique is revealing blood pressure you will know how to see through the variability of each technique and how to read the changes in BP.

Acknowledgments. This research did not receive any specific grant from funding agencies in the public, commercial, or not-for-profit sectors.

\section{References}

1. Accardi MV, Troncy E, Abtout S, Ascah A, Maghezzi S, Authier S (2016). Rat cardiovascular telemetry: Marginal distribution applied to positive control drugs. Journal of pharmacological and toxicological methods 81:120-127.

2. Alam MA, Parks C, Mancarella S (2016). Long-term blood pressure measurement in freely moving mice using telemetry. Journal of visualized experiments (111). doi: 10.3791/53991.

3. Araghi A, Bander JJ, Guzman JA (2006). Arterial blood pressure monitoring in overweight critically ill patients: invasive or noninvasive?. Critical Care, 10(2): R64.

4. Bazil MK, Krulan C, Webb RL (1993). Telemetric monitoring of cardiovascular parameters in conscious spontaneously hypertensive rats. Journal of cardiovascular pharmacology 22(6):897-905.

5. Behr-Roussel D, Oudot A, Compagnie S, Gorny D, Le Coz O, BernabeJ,GiulianoF (2008).Impactofalong-term sildenafil treatment on pressor response in conscious rats with insulin resistance and hypertriglyceridemia. American journal of hypertension 21(11):1258-1263.

6. Bogdan SA, Luca V, Pestean C, Ober C, Bel L, Codea RA (2017). Measurement of mean arterial pressure by carotid artery cannulation in isoflurane anesthetized wistar rats. Bulletin USAMV Veterinary Medicine, 74(1):98-104.

7. Braga VA, Prabhakar NR (2009). Refinement of telemetry for measuring blood pressure in conscious rats. Journal of the American Association for Laboratory Animal Science 48(3): 268-271.

8. Braga VA, Burmeister MA (2011). Applications of telemetry in small laboratory animals for studying cardiovascular diseases. Modern Telemetry. IntechOpen.

9. Brockway BP, Mills PA, Azar SH (1991). A new method for continuous chronic measurement and recording of blood pressure, heart rate and activity in the rat via radiotelemetry. Clinical and Experimental Hypertension. Part A: Theory and Practice 13(5):885-895.

10. BuÑag RD, Butterfield J (1982). Tail-cuff blood pressure measurement without external preheating in awake rats. Hypertension 4(6):898-903.

11. Burkitt Creedon J.M. (2012). Indirect Blood pressure measurement, NAVC Clinician's Breif, Procedures Pro Cardiology, p26-30.

12. Daugherty A, Rateri DL, Hong L, Balakrishnan A (2009). Measuring blood pressure in mice using volume pressure recording, a tail-cuff method. Journal of visualized experiments: 15;(27). pii: 1291. doi: 10.3791/1291

13. De Wolff MH, Leather HA, Wouters PF (1999). Effects of tramadol on minimum alveolar concentration (MAC) of isoflurane in rats. British Journal of Anaesthesia 83(5):780783.

14. Durham HE (2005). Arterial blood pressure measurement, Monitoring and Nursing, vol 26, No5.

15. Erken HA, Erken G, Genç O (2013). Blood pressure measurement in freely moving rats by the tail cuff method. Clinical and Experimental Hypertension 35(1):11-15. 
16. Ettinger SJ, Edward CF, Etienne C (2017). Textbook of veterinary internal medicine, $8^{\text {th }}$ edition (2017), Vol 1 , Elsevier, p. 377-381

17. Faunt K (2008). Anesthesia for the pet practitioner. Anesthesia for the pet practitioner., (Ed. 2). p.21-23.

18. Feng J, Fitz Y, Li Y, Fernandez M, Puch IC, Wang D, Solomon SB (2015). Catheterization of the carotid artery and jugular vein to perform hemodynamic measures, infusions and blood sampling in a conscious rat model. Journal of visualized experiments (95). doi: 10.3791/51881

19. Feng M, Whitesall S, Zhang Y, Beibel M, Alecy LD, DiPetrillo $\mathrm{K}$ (2008). Validation of volume-pressure recording tailcuff blood pressure measurements. American journal of hypertension 21(12):1288-1291.

20. Filipas M, Vettorato E (2018), Manage hypotension under general anaesthesia. Companion, BSAVA Issue 8, p. 10 17.

21. Hartley CJ, Taffet GE, Reddy AK, Entman ML, Michael LH (2002). Noninvasive cardiovascular phenotyping in mice. ILAR Journal 43(3):147-158.

22. Hentschel S (2008). Modeling and simulation of blood pressure in rats. Master's Thesis.

23. Howatt DA, Balakrishnan A, Lu H (2017). Blood pressure Measurment: Kent Coda 8 (Tail-Cuff), Protocols, vol 10

24. Huetteman DA, Bogie H (2009). Direct blood pressure monitoring in laboratory rodents via implantable radio telemetry. In Cardiovascular Genomics (pp. 57-73). Humana Press, Totowa, NJ.

25. Imai A, Steffey EP, Farver TB, Ilkiw JE (1999). Assessment of isoflurane-induced anesthesia in ferrets and rats. American Journal of Veterinary Research 60(12):1577-1583.

26. Jespersen B, Knupp L, Northcott CA (2012). Femoral arterial and venous catheterization for blood sampling, drug administration and conscious blood pressure and heart rate measurements. Journal of Visualized Experiments (59). pii: 3496. doi: 10.3791/3496.

27. Jin YX, Kim HR, Kim JH, Park KY, Kim SY (2013). Effects of mineral-rich salt intake on the serum and blood pressure of Dahl salt-sensitive rats. Journal of Biomedical Research 14(4):212-219.

28. Kramer K, Kinter LB (2003). Evaluation and applications of radiotelemetry in small laboratory animals. Physiological genomics 13(3):197-205.

29. Kramer K, Remie R (2005). Measuring blood pressure in small laboratory animals. In Hypertension (pp. 51-62). Humana Press.

30. Kregel KC, Wall PT, Gisolfi CV (1988). Peripheral vascular responses to hyperthermia in the rat. Journal of applied physiology 64(6):2582-2588.

31. Krinke GJ (2000). The laboratory rat. Elsevier. p463-479

32. Kurtz TW, Griffin KA, Bidani AK, Davisson RL, Hall JE (2005). Recommendations for blood pressure measurement in humans and experimental animals: part 2: blood pressure measurement in experimental animals. A statement for professionals from the Subcommittee of Professional and Public Education of the American
Heart Association Council on High Blood Pressure Research. Arteriosclerosis, Thrombosis, and Vascular Biology 25(3), e22-e33.

33. Lin HT, Shiou YL, Jhuang WJ, Lee HC (2019). Simultaneous Electrocardiography Recording and Invasive Blood Pressure Measurement in Rats. Journal of Visualized Experiments (143), e59115.

34. Malkoff J. (2005). Non-invasive blood pressure for mice and rats, Animal Lab News, Kent Scientific Corporation, $1-12$.

35. Meredith A (2015). BSAVA Small Animal Formulary: Part B: Exotic Pets, John Wiley \& Sons.

36. Michalak GJ, Schwartz JA, Goodrich GP, O’Neal DP (2010). Three-wavelength murine photoplethysmography for estimation of vascular gold nanorod concentration. Optics Express 18(25):26535-26549.

37. Ng KG, Small CF (1993). Changes in oscillometric pulse amplitude envelope with cuff size: implications for blood pressure measurement criteria and cuff size selection. Journal of biomedical engineering 15(4):279282.

38. Nguyen A, Armstrong EA, Yager JY (2015). Unilateral Common Carotid Artery Ligation as a Model of Perinatal Asphyxia: The Original Rice-Vannucci Model. In Animal Models of Neurodevelopmental Disorders (pp. 1-13). Humana Press, New York, NY.

39. Neuwelt EA, Kroll RA (1994). Osmotic Blood--Brain Barrier Modification: Increasing Delivery of Diagnostic and Therapeutic Agents to the Brain. In Methods in Neurosciences (Vol. 21, pp. 52-67). Academic Press.

40. Oriana D, Blood Pressure Measurement (October 2018), Veterinary Team Brief, 23-27

41. Ordodi VL, Mic FA, Mic AA, Toma O, Sandesc D, Paunescu $\mathrm{V}$ (2005). A simple device for invasive measurement of arterial blood pressure and ECG in the anesthesized rat. Timisoara Med J 55(8):35-7.

42. Oudot A, Behr-Roussel D, Compagnie S, Caisey S, Le Coz O, Gorny D, Giuliano F (2009). Endothelial dysfunction in insulin-resistant rats is associated with oxidative stress and COX pathway dysregulation. Physiological research 58(4):499-509.

43. Pacher P, Nagayama T, Mukhopadhyay P, Bátkai S, Kass DA (2008). Measurement of cardiac function using pressurevolume conductance catheter technique in mice and rats. Nature protocols, 3(9):1422.

44. Parasuraman S, Raveendran R (2012). Measurement of invasive blood pressure in rats. Journal of Pharmacology and Pharmacotherapeutics 3(2):172.

45. Patten GS, Abeywardena MY (2017). Effects of antihypertensive agents on intestinal contractility in the spontaneously hypertensive rat: angiotensin receptor system downregulation by losartan. Journal of Pharmacology and Experimental Therapeutics 360(2): 260-266.

46. Reddy A.K, Taffet GE, Madala S, Michael LH, Entman ML, Hartley CJ (2003). Noninvasive blood pressure measurement in mice using pulsed Doppler ultrasound. Ultrasound in medicine \& biology 29(3):379-385. 
47. Rehman S, Nelson VL (2018). Blood Pressure Measurement. In: StatPearls [Internet]. Treasure Island (FL): StatPearls from: https://www.ncbi.nlm.nih.gov/ books/NBK482189 accessed in 10.01.2019

48. Tran CHT, Gordon GR (2015). Acute two-photon imaging of the neurovascular unit in the cortex of active mice. Frontiers in cellular neuroscience 9:11. doi: 10.3389/fncel.2015.00011.

49. Tranquilli WJ, Thurmon JC, Grimm KA (2013). (Eds.) Lumb and Jones' veterinary anesthesia and analgesia. John Wiley \& Sons.

50. Wang Y, Cong Y, Li J, Li X, Li B, Qi S (2013). Comparison of invasive blood pressure measurements from the caudal ventral artery and the femoral artery in male adult sd and wistar rats. PloS one, 8(4), e60625.

51. Ward M, Langton JA (2007). Blood pressure measurement, Continuing Education in Anaesthesia, Critical Care \& Pain 7 (4):122-128.

52. Whitesall SE, Hoff JB, Vollmer AP, D’Alecy LG (2004). Comparison of simultaneous measurement of mouse systolic arterial blood pressure by radiotelemetry and tail-cuff methods. American Journal of Physiology-Heart and Circulatory Physiology 286(6):H2408-15.

53. Wilde E, Aubdool AA, Thakore P, Baldissera JL, Alawi KM, Keeble J, Brain SD (2017). Tail-cuff technique and its influence on central blood pressure in the mouse. Journal of the American Heart Association 6(6):e005204.

54. Will N (2003). Anaesthesia for Pet Practitioner, Banfield, USA 68-70, 90-91 p

55. Tranquilli WJ, Thurmon JC, Grim KA (2007). Lumb\&Jones's Veterinary Anaesthesia and Analgesia. $4^{\text {th }}$ ed. Blackwell Publishing 355-383, 533-547 p

56. Wilson TE, Crandall CG (2011). Effect of thermal stress on cardiac function. Exercise and sport sciences reviews 39(1):12.

57. Wingfield WE, Raffe MR (2002). The Veterinary ICU Book, Teton New Media, Jackson Hole, Wyoming, 266-280

58. https://www.harvardapparatus.com/rat-catheters.html

59. https://www.instechlabs.com/products/catheters/ratcarotid

60. https://www.wpi-europe.com/products/animalphysiology/blood-pressure-measurement/ii-mrbp-raspx

61. https://www.iitcinc.com/3man.html accessed in 16.04.2019

62. http://www.iitcinc.com/Mouseratbloodpressure.html

63. http://www.itsbio.co.kr/main/gmb_board_view. php? page $=1 \&$ no $=444 \&$ search $=\&$ page_no $=1$

64. https://www.kentscientific.com/products/coda-highthroughput-system/ 